\title{
Siah Ubiquitin Ligases Modulate Nodal Signaling during Zebrafish Embryonic Development
}

\author{
Nami Kang ${ }^{1,3}$, Minho Won ${ }^{2,3}$, Myungchull Rhee ${ }^{1, *}$, and Hyunju Ro ${ }^{1, *}$
}

Siah2 is a zebrafish homologue of mammalian Siah family. Siah acts as an E3 ubiquitin ligase that binds proteins destined for degradation. Extensive homology between siah and Drosophila Siah homologue (sina) suggests their important physiological roles during embryonic development. However, detailed functional studies of Siah in vertebrate development have not been carried out. Here we report that Siah2 specifically augments nodal related gene expression in marginal blastomeres at late blastula through early gastrula stages of zebrafish embryos. Siah2 dependent Nodal signaling augmentation is confirmed by cell-based reporter gene assays using 293T cells and 3TPluciferase reporter plasmid. We also established a molecular hierarchy of Siah as a upstream regulator of FoxH1/Fast1 transcriptional factor in Nodal signaling. Elevated expression of nodal related genes by overexpression of Siah2 was enough to override the inhibitory effects of atv and Ift2 on the Nodal signaling. In particular, E3 ubiquitin ligase activity of Siah2 is critical to limit the duration and/or magnitude of Nodal signaling. Additionally, since the embryos injected with Siah morpholinos mimicked the atv overexpression phenotype at least in part, our data support a model in which Siah is involved in mesendoderm patterning via modulating Nodal signaling.

\section{INTRODUCTION}

Siah is the mammalian homologue of the protein encoded by Drosophila seven in absentia (sina) that is required for proper R7 photoreceptor development (Carthew and Rubin 1990; Hu et al., 1997; Li et al., 1997; Tang et al., 1997). Various studies showed that both SINA and Siah act as E3 ubiquitin ligase for

\footnotetext{
${ }^{1}$ Department of Biological Sciences, College of Bioscience and Biotechnology, Chungnam National University, Daejeon 305-764, Korea, ${ }^{2}$ Program in Genomics of Differentiation, Eunice Kennedy Shiver National Institute of Child Health and Human Development, National Institutes of Health, Bethesda, Maryland, USA, ${ }^{3}$ These authors contributed equally to this work.

${ }^{*}$ Correspondence: mrhee@ cnu.ac.kr (MR); rohyunju@cnu.ac.kr (HR)
}

Received 18 February, 2014; revised 27 March, 2014; accepted 31 March, 2014; published online 14 May, 2014

Keywords: antivin/Lft1, body patterning, FoxH1/Fast1, Nodal, Siah2, zebrafish target proteins degradation (House et al., 2009; Qi et al., 2013; Wong and Möller, 2013). Since enforced expression of Siah1 causes cellular growth arrest or can be pro-apoptotic (Matsuzawa et al., 1998), Siah proteins were initially reported as tumor suppressors. We also previously reported that transient expression of zebrafish Siah2 in Bosc 23 and HeLa cells induced cell cycle arrest via inhibiting $\mathrm{G}_{2} / \mathrm{M}$ phase transition, suggesting that vertebrate Siah family may share their cellular functions (Ro et al., 2003). However, the tumor suppressor function of Siah has been controversial, given the lack of somatic SIAH mutation in human cancers (Medhioub et al., 2000). In addition, several reports support an oncogenic role of Siah proteins in animal cancer model systems (Wong and Möller, 2013)

Vertebrate body patterning is initiated during gastrulation as endoderm, mesoderm and ectoderm are induced along the dorsoventral (DV) and anteroposterior (AP) axes. Fgf, Wnt and Nodal signals initiate early dorsoventral pattern in zebrafish embryos (Schier and Talbot, 2005). After fertilization the dorsal determinants are translocated to the dorsal side through the subcortical microtubules. The dorsal determinants lead to the activation of maternal Wnt signaling. Maternal Wnt, especially Wnt8a in the case of zebrafish activates $\beta$-catenin, a transcriptional activator, to regulate expression of several genes important for the early embryonic body patterning (Huelsken and Birchmeier, 2001; Lu et al., 2011). Gore et al. (2005) proposed that Squint (Sqt/Ndr1), one of the Nodal-related proteins, acts as a potent dorsal determinant through the differential localization as early as four cells stage. Previous works showed that Siah1 functions as a negative regulator of the Wnt signaling by down-regulating $\beta$-catenin stability (Liu et al., 2001; Matsuzawa and Reed, 2001). Siah2 up-regulates Fgf downstream signaling by destructing Sprouty (a Fgf-dependent ERK inhibitor) in a phosphorylation-independent manner while Siah1 does not (Nadeau et al., 2006; Qi et al., 2008). It thus appears that Siah family plays critical roles in vertebrate body axis formation by modulating various signaling pathways.

Several genetic studies and overexpression experiments indicated that nodal related genes are required for the DV and AP patterning (Feldman et al., 1998; Langdon and Mullins, 2011). Nodal belongs to the TGF- $\beta$ superfamily that transmits signals through heterodimeric type I and type II Activin like receptors (Thisse and Thisse, 1999; Whitman, 2001). Nodal signals lead to formation of a protein complex comprising Smad2/4 and FoxH1 (Fast1; forkhead transcriptional factor) to activate downstream target genes (Attisano et al., 2001; Chen et al., 1997). In zebrafish, two nodal-related genes, squint (sqt/ndr1) 
and cyclops (cyc/ndr2) are required for the axial and trunk mesoderm formation as well as endoderm induction (Langdon and Mullins, 2011; Rebagliati et al., 1998). Nodal signaling is also critical for neural patterning, as sqt;cyc double mutant or oneeyed pinhead (MZoep) mutant embryos lacking an EGF-CFC co-receptor essential for Nodal signaling developed expansion of anterior neural fate and loss of trunk spinal cord (Feldman et al., 1998; Gritsman et al., 1999). Erter et al. (2001) reported that Wnt8 expression is locally down-regulated at the dorsal midline, but persists ventrolaterally in sqt, cyc double mutants or Antivin (Atv, a potent nodal antagonist) overexpressed embryos. Since enforced expression of Nodal in the anterior neuroectoderm induces notochord formation at the expense of forebrain (Thisse et al., 2000), it is thus conceivable that Nodal acts as anteriorizing transformer signal for posterior neuroectoderm via directly or indirectly up-regulating zygotic Wnt8 expression in tissues outside of the organizer, specifically the lateral/paraxial mesoderm.

We have previously isolated and characterized a zebrafish Siah and designated it Siaz (renamed to Siah2l following ZFIN designation. But we will call the Siah2l as Siah2 in this report to avoid confusion) (Ro et al., 2003; 2005). In this report, we demonstrated that Siah as a critical intracellular Nodal modulator acts on upstream of Fast1/FoxH1 transcriptional factor for the maintenance of Nodal homeostasis. We revealed that the functional RING domain of Siah2 is exclusively required for antagonizing the function of Nodal inhibitors. Positive roles of Siah for Nodal signaling were reinforced by gene depletion experiments. Knocking-down of Siah1 and Siah2 with specific morpholinos (MO) induced the morphants partially mimicking the morphology of Nodal defective mutants. Collectively, these results clarify the roles of Siah family in Nodal signaling.

\section{MATERIALS AND METHODS}

Fish maintenance

Fish and embryos were maintained essentially as described in the zebrafish book (Westerfield, 1995).

Cell culture and transfection

293T cells were grown in Dulbecco's Modified Eagle's medium (DMEM) supplemented 10\% fetal bovine serum (FBS). Cells in culture dishes were transfected with various plasmid constructs (pFlag/Siah2, pFlag/Siha2 Mu, pcGlobin2/Siah2, pcGlobin2/Fast1, pcGlobin2/Fast1-SID) using FuGene6 transfection reagent (Roche). After $24 \mathrm{~h}$, cells were harvested and then the cell pellet was used for further assay.

\section{GST-pull down and Western blotting}

In vitro binding assay was performed by mixing embryonic lysates after injecting GST/ $\beta$-catenin or GST/ $\beta$-catenin mutant (stabilized $\beta$-catenin) RNA with $10 \mu \mathrm{l}$ of glutathione-Sepharose 4B (Pharmacia Biotech, Sweden) in total $800 \mu$ l volume of NP-40 lysis buffer $\left(150 \mathrm{mM} \mathrm{NaCl}, 0.02 \% \mathrm{NaN}_{3}, 25 \mu \mathrm{M}\right.$ PMSF, $1 \mathrm{mM}$ EDTA, $10 \mathrm{mM}$ Tris-Cl, pH 7.2, $0.5 \% \mathrm{NP}-40$ ) at $4^{\circ} \mathrm{C}$ for $4 \mathrm{~h}$ agitation. Precipitates were washed at least three times with ice-cold NP-40 buffer ( $1 \mathrm{ml}$ ) and were analyzed by Western blotting. After separated in SDS-PAGE (10\%) and transferred into PVDF membrane, the membrane was incubated for $1 \mathrm{hr}$ under the blocking solution ( $2 \%$ skim milk in PBS with $0.05 \%$ Tween-20) at room temperature and then were incubated with anti-Flag (Sigma), anti- $\beta$-catenin (Sigma) and anti-GST (Ab Frontier) antibody diluted in blocking solution for $1 \mathrm{~h}$, followed by incubation with HRP-conjugated secondary antibody (Bio-Rad, 1:1000) for $1 \mathrm{~h}$ at room temperature. The detection was performed using Prona ${ }^{\mathrm{TM}}$ ECL Ottimo detection system (TransLab).

Luciferase assay

We co-transfected 3TP-Luc reporter construct $(0.5 \mu \mathrm{g})$ with indicated DNAs into 293T cells using calcium phosphate precipitation technique. Total amount of transfected DNA was equalized with control vectors without inserts. Transfected 293 cells were treated with TGF- $\beta(1 \mathrm{ng} / \mathrm{ml})$ for $24 \mathrm{~h}$, and lysed for luciferase assay using the Dual Luciferase system (Promega).

\section{Whole mount in situ hybridization}

Antisense riboprobes were constructed using appropriate RNA polymerase following the instructions (Ambion). In situ hybridization analysis followed the protocol of Westerfield (1995) with small modification. Proteinase $\mathrm{K}$ treatment $(10 \mu \mathrm{g} / \mathrm{ml})$ was performed for 3 to 10 min depending on the stages of embryos. The hybridized probes were detected using pre-absorbed antidigoxigenin-AP Fab fragments (Roche) diluted (1:2000) in blocking solution (PBS, $0.1 \%$ Tween-20, 5\% sheep serum). After 3-8 h staining, embryos were mounted in a 2:1 mixture of benzylbenzoate:benzylalcohol and examined under the microscope.

\section{mRNA and MO microinjection}

For the microinjection, cDNA constructs were subcloned into the pcGlobin2 vector (Ro et al., 2004b). mRNAs for injection were synthesized from the vector constructs linearized with the appropriate restriction enzymes, using the mMESSAGE mMACHINE T7 kit (Ambion Inc.) according to the manufacturers instruction. After purification as following the manufacturers recommendation, mRNAs were dissolved in diethylpyrocarbonate (DEPC)-treated $0.1 \mathrm{M} \mathrm{KCl}$. Before injection, the mRNAs were diluted to various concentrations and $1 \mu \mathrm{l}$ of mRNA was used to inject approximately 400 embryos. The mRNA and MOs were pressure injected into the yolk of 1-2 cell stage of embryos and the injected embryos were raised in 1/3 Ringers solution $\left(39 \mathrm{mM} \mathrm{NaCl}, 0.97 \mathrm{mM} \mathrm{KCl}, 1.8 \mathrm{mM} \mathrm{CaCl}_{2}, 1.7 \mathrm{mM}\right.$ Hepes, pH 7.2). Injections were performed three to four times to pool the data. To reduce the p53-dependent off-targeting effects of siah MOs, we coinjected the MOs with a p63 MO (Robu et al., 2007). MO sequences were: siah1 translate blocker, 5'-CTGGCGACTCATITCTTCGTCCATA-3'; siah2 translate blocker, 5'-TCGACGTTTGATGGTGTAAAACCCC-3'.

\section{RT-PCR}

Total RNA was isolated from zebrafish embryos using easyBLUE $^{\text {TM }}$ (INTRON, Inc.) and $1 \mu \mathrm{g}$ of RNA was used for RT-PCR. sqt and $\beta$-actin specific forward and reverse primers were used for this reaction. sqt, 5'-CAAGCagaaccgggcaaagacgtcc- $3^{\prime}$ (forward) and 5'-gtggcagccgcattctgcaacaacc-3' (reverse); $\beta$ actin, 5'-gaggagcaccccgtcctgctcac-3' (forward) and 5'-gatggct ggaacagggcctctgg-3' (reverse)

\section{Site directed mutagenesis}

For the amino acids substitution of Siah2 H107A and C110A, mega-primer was generated by PCR using Pfu polymerase (forward primer; 5'-atgagccgtccgtcctctgcgg- ${ }^{\prime}$, reverse primer; 5 '- gacactggttagccaccagagccccagcctggc-3', Underlines indicate mutated sequences). Generated mega-primer together with another primer (5'-ttagcacatagagatggtcac- $\left.3^{\prime}\right)$ was used for the amplification of full length mutated Siah2. Amplified PCR product was subcloned into T-vector (pGEM-T Easy Vector Systems; Promega). 

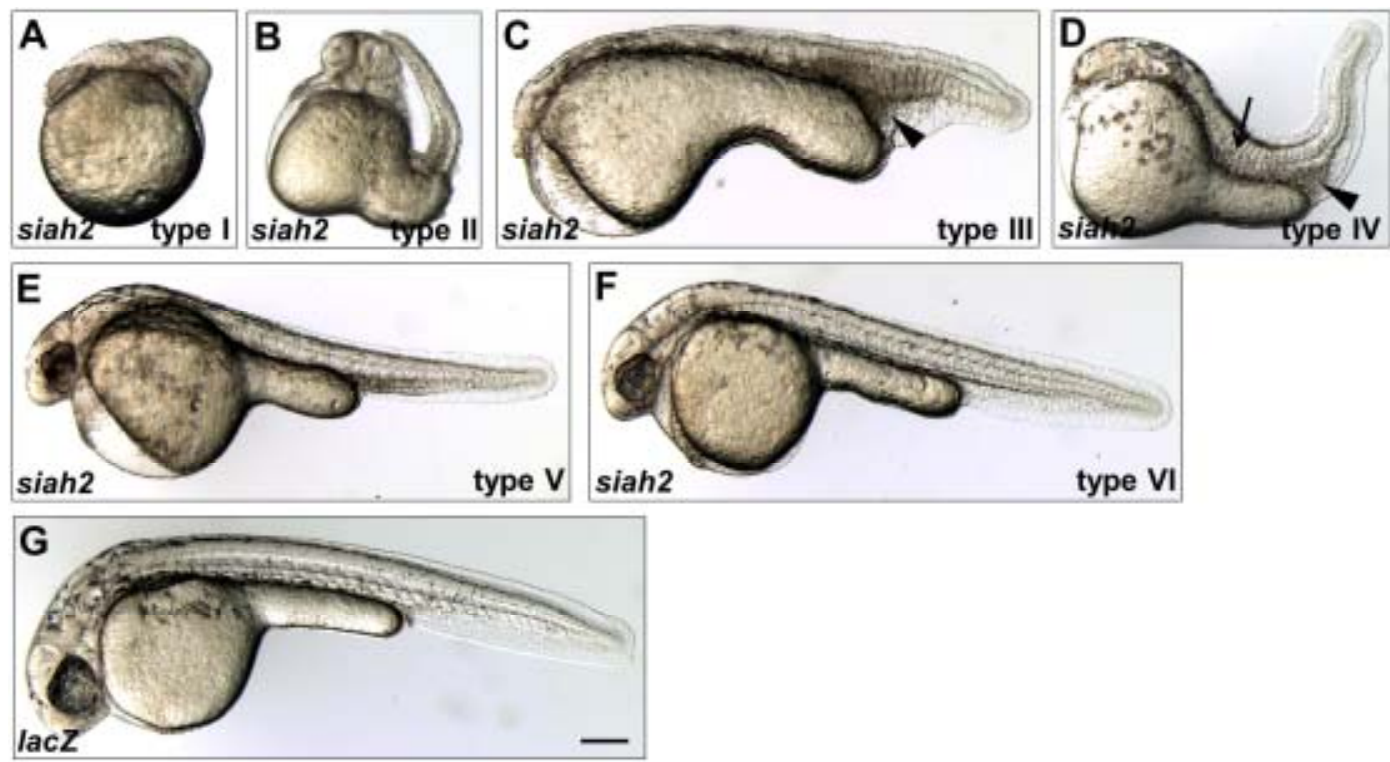

Fig. 1. Injection of siah2 induces various embryonic defects. Injected embryos were examined using microscopy at $36 \mathrm{hpf}$ (larval view). (A-F) Phenotypes induced by Siah2 overexpression; type I, head rudiments combination with absence of axial structure and truck; type II, brain defects, degenerated truck and curled tail structure; type III, extra cells in the tail (arrowhead) and reduced head structure; type IV, partially degenerated notochord (arrow), forebrain defects and extra cell mass in tail (arrowhead); type V, small head and slightly reduced yolk sac; type $\mathrm{VI}$, morphologically indistinguiahable from uninjected embryo. (G) Control embryos injected with $500 \mathrm{pg}$ lacZ mRNA. Scale bar, $200 \mu \mathrm{m}$.

\section{RESULTS}

Siah2 overexpression disturbs early dorso-ventral body pattern

siah2 is maternally expressed and its zygotic expression starts at the mid-blastula transition (MBT) in all blastomeres (Ro et al., 2003). To determine the embryonic function of Siah2, siah2 RNA $(500 \mathrm{pg})$ was microinjected into the yolk of 1-2 cell stage embryos. When Siah2 was vastly expressed, embryos showed severely dorsalized (Figs. $1 A$ and $1 B, n=145 / 305,47.5 \%$ ) or ventralized phenotypes (Figs. 1C and 1D, $n=63 / 305,21 \%$ ). Those opposite embryonic defects were reminiscent of the embryos injected with smad7 RNA (Pogoda and Meyer, 2002). Pogoda and Meyer (2002) showed that overexpression of Smad7 induced phenotypes similar to that of MZoep mutant or bmp7 mutant (snh). The opposite defects caused by Smad7 overexpression might be due to the inhibitory effects of Smad7 on both Nodal and Bmp signaling pathways. To examine if Siah2 modulates two separate signaling pathways for the DV body patterning, we initially analyzed if Siah2 down-regulates $\beta$-catenin stability as mammalian Siah1 does (Liu et al., 2001; Matsuzawa and Reed, 2001). $\beta$-catenin overexpression caused complete secondary axis formation (Fig. $2 A, n=54 / 109,49.5 \%$ ) as well as ectopic expression of $g s c$ (Fig. $2 \mathrm{C}, \mathrm{n}=41 / 44,93.2 \%)$. When Siah2 was co-expressed with $\beta$ catenin, the secondary axis formation was nearly abolished (Fig. $2 B, n=19 / 229,8 \%$ of embryos formed secondary axis), and ventrolateally expanded gsc expression was suppressed (Fig. 2E, n $=9 / 44$, only $20 \%$ of embryos showed expanded gsc expression). In contrast, $\mathrm{N}$-terminal Ring deleted or $\mathrm{C}$-terminal protein-protein interacting domain deleted form of Siah2 (Ro et al., 2004a) did not suppress the ectopic gsc expression (Fig. $2 \mathrm{~F}, \mathrm{n}=40 / 44$, $91 \%$; and $2 \mathrm{G}, \mathrm{n}=34 / 39,87 \%$, Fig. $2 \mathrm{H}$ ). Since Siah1 destructs $\beta$ catenin independent of GSK-3 (Liu et al., 2001; Matsuzawa and
Reed, 2001), we wondered whether Siah2 destabilizes $\beta$-catenin in similar manner. We injected RNA encoding GST-tagged wild type (GST- $\beta$-cat) or phosphorylation resistant $\beta$-catenin (GST- $\beta$ cat $\mathrm{Mu}$ ) alone or together with siah2 into 1-2 cell stage embryos, and then detected exogenously supplemented $\beta$-catenin using anti- $\beta$-catenin antibody or anti-GST antibody after pull-downed using glutathione-sepharose beads. Expression of both WT and phosphorylation resistant $\beta$-catenin were remarkably reduced by the co-expression of Siah2 (Figs. 2l and 2J) indicating that Siah2 may induce embryonic ventralization by down-regulating maternally derived Wnt signaling through the destruction of $\beta$-catenin.

Interestingly, squint (sqt) expression increased in the presumptive mesendoderm after siah2 RNA injection as the expression area expanded from the blastoderm margin toward the animal pole at $40 \%$ epiboly (Figs. 3A-3D; embryos with ectopic sqt expression: $40 \%$ epiboly: $55 \%, n=40$; germ ring: $48 \%$ : $n=44)$. cyclops (cyc) expression was also increased in Siah2 overexpressed embryos (Figs. 3E and 3F; embryos with ectopic cyc expression at $40 \%$ epiboly: $44 \%, n=45$ ). The dorsal specific expression domain of $c y c$ was largely expanded ventrolaterally in the siah2 injected embryos (Figs. $3 \mathrm{G}$ and $3 \mathrm{H}$; embryos with ectopic cyc expression at germ ring: $41 \%, n=41$ ). These results further support the previous report that dorsalization can be induced by the augmented Nodal signaling (Erter et al., 1998). It is also plausible to consider that the Siah2 dependent embryonic dorsalization, at least to some extent, resulted from dampened zygotic Wnt signaling which is important for the initial specification and maintenance of ventro-posteror mesodermal tissues. Thus, the opposite DV defective phenotypes caused by Siah2 overexpression could be induced by biased diminution of maternal or zygotic Wnt signaling together with elevated induction of nodal related genes. 

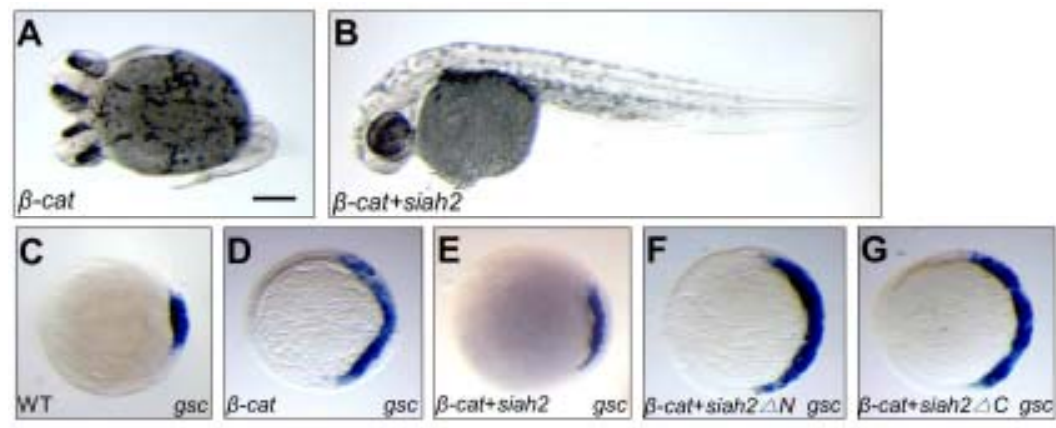

H

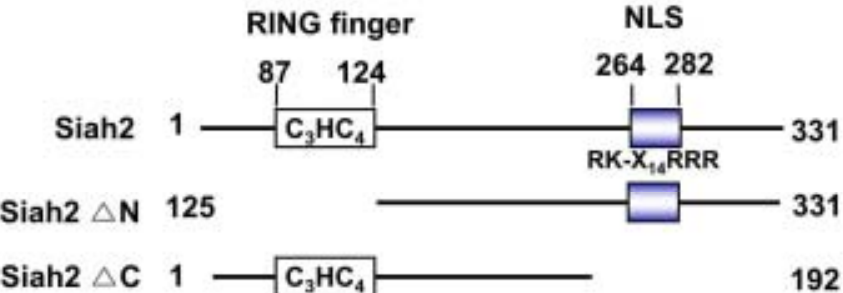

Siah2 $\triangle \mathrm{C} 1-\mathrm{C}_{3} \mathrm{HC}_{4}$

I
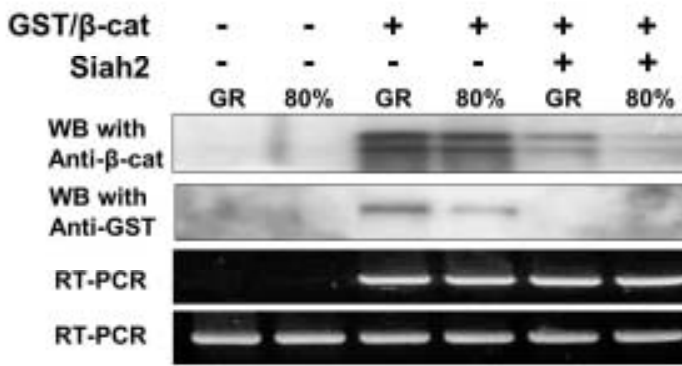

$\leftarrow$ GST/ $\beta$-cat

$\leftarrow \beta$-actin
J

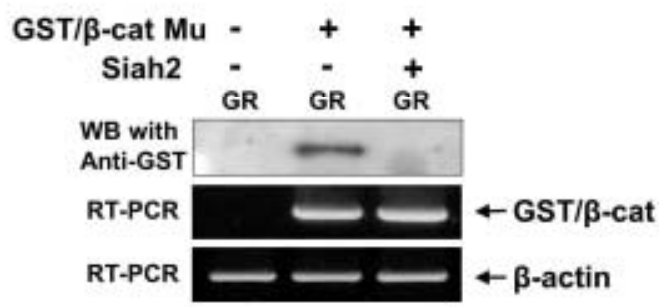

Fig. 2. Siah2 overexpression counteracts $\beta$ catenin-induced secondary axis formation via down-regulating $\beta$-catenin. Microinjected genes were indicated in the lower left corner of each panel. (A, B) Zebrafish embryos at $36 \mathrm{hpf}$. (A) WT embryo was injected with $225 \mathrm{pg}$ of $\beta$-catenin mRNA. Overexpression of $\beta$-catenin induced secondary body axis. (B) Co-injection of $\beta$-catenin (225 pg) and siah2 (100 pg) RNA. Siah2 suppressed hyper-expression of gsc by WT or stabilized $\beta$-catenin. (C, D) gsc expression in the dorsal hypoblast of WT embryos (C) was activated by $\beta$-catenin (225 pg) overexpression (D). (E) hyper- expression of $g s c$ by $\beta$-catenin was suppressed in the embryos co-injected with siah2 (100 pg). ( $F, G) N$-terminal (Siah2 $\Delta N)$ or C-terminal deletion (Siah2 $\Delta \mathrm{C}$ ) of Siah2 failed to suppress hyper-expression of $g s c$ by $\beta$-catenin. $(H)$ Diagram represents $W T$ and deletion constructs of Siah2. (I, J) Siah2 down-regulates $\beta$-catenin in a GSK-3 $\beta$ mediated phosphorylation independent manner. GST-pull down of GST- $\beta$-catenin with glutathione-Sepharose agarose bead. (I) Embryo extracts from germ ring and $80 \%$ epiboly stage injected with GST- $\beta$-catenin $(225 \mathrm{pg})$ alone or combination with siah2 $(100 \mathrm{pg})$. Blots were probed with rabbit polyclonal antibody against $\beta$-catenin or anti-GST antibody. (J) Embryo extracts from germ ring and $80 \%$ epiboly stage injected with GST- $\beta$ catenin $\mathrm{Mu}(225 \mathrm{pg})$ alone or together with siah2 (100 pg). Blots were detected with antiGST antibody. Note that injected GST- $\beta$ catenin or GST- $\beta$-catenin Mu mRNAs were not affected by Siah2 overexpression. Ethidium bromide staining of the $\beta$-actin RT-PCR products verifies internal control for the reactions. Scale bar, $200 \mu \mathrm{m}$.
Siah2 overexpression restores lefty-induced mesendodermal defects

To ask if the augmented nodal related genes expression by Siah2 involves in mesendoderm specification, we co-injected $100 \mathrm{pg}$ of siah2 RNA with $25 \mathrm{pg}$ of atv (lefty1) or Ift2 (lefty2) RNA. Overexpression of atv or Ift2, the Nodal antagonists, induces severe mesendodermal defects silftmilar to the MZoep or sqt/cyc double mutant phenotype (Bisgrove et al., 1999; Schier and Talbot, 2001). Atv and Lft2 compete with Nodal for binding to the type I and II Activin receptor complex (Thisse and Thisse, 1999). As shown in Figs. 4B, 4D, 4Q, and 4S, blocking of Nodal signaling with atv or Ift2 overexpression caused severe embryonic defects, such as malformation of embryonic shield, and incomplete mesendoderm-derived tissue formation manifested by fused eyes, no visible notochord and column-like somites in reduced numbers. In contrast, in embryos coinjected with siah2 RNA development of the embryonic shield, notochord, somites was restored (Figs. 4C, 4E, and 4Q-4T).
We confirmed the antagonizing effects of Siah2 on Atv/Lft2 by analyzing expression patterns of shh and col2a1, marker genes for floor plate and notochord, respectively. In the embryos injected with atv/ft2 RNA (25 pg) alone, the transcripts of both shh and col2a1 were nearly abolished at 24 hpf (Figs. 5A, $5 \mathrm{C}, 5 \mathrm{D}$, and $5 \mathrm{~F})$. But in the embryos co-injected with atv (25 pg) and siah2 RNA (100 pg), expression of shh in the floor plate and col2A1 in the axial mesoderm was restored (Figs. 5G and $5 \mathrm{I}$ ). The somite defects manifested by severely compromised myoD expression in atv overexpressed embryos were also rescued by exogenous of Siah2 (Figs. 5B, 5E, and $5 \mathrm{H}$ ). In addition, the enlarged forebrain at the expense of diencephalon marked with $d l \times 2$ and emx 1 staining in atv overexpressing embryos was ameliorated by increased Siah2 expression level (Figs. 5B, 5C, 5E, 5F, 5H, and 5I). We obtained similar results with Ift2 and siah2 RNA injection (data not shown).

To investigate whether the Siah2 reconstituted nodal related gene expression in the developing mesendodermal cells in 

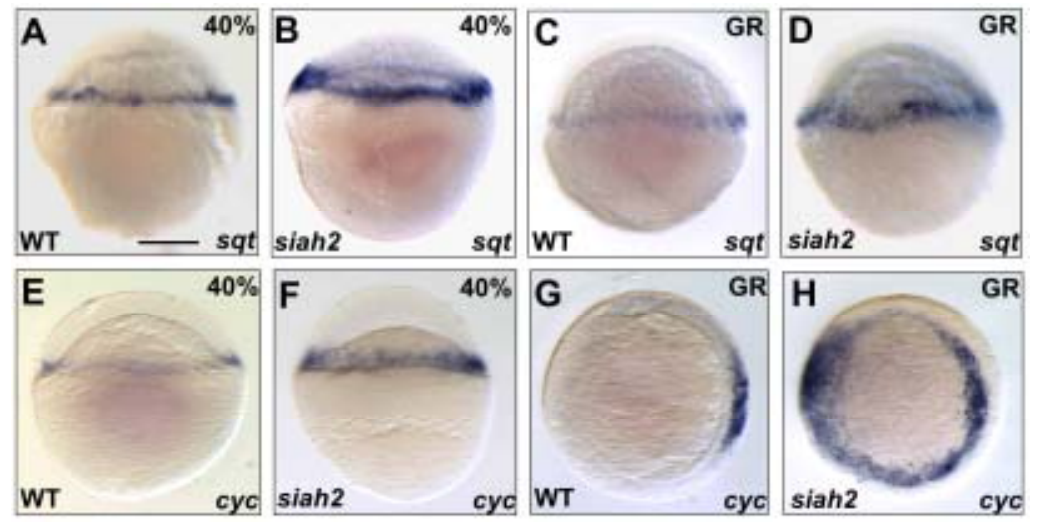

Fig. 3. Siah2 up-regulates nodal-related genes. Expression of the marker genes was analyzed by in situ hybridization: sqt in WT $(\mathrm{A}, \mathrm{C})$ and siah2 injected (B, D) embryos at $40 \%$ epiboly $(A, B)$ and germ ring $(C, D)$. cyc in WT $(E, G)$ and siah2 injected $(F, H)$ embryos at $40 \%$ epiboly $(E, F)$ and germ ring $(G, H)$. Embryos are shown in the following orientations: lateral view with dorsal to the right (A-F), animal pole view with dorsal to the right $(\mathrm{G}, \mathrm{H})$. WT or siah2 injected embryos are indicated in the lower left corner of each panel; marker genes for expression analysis in the lower right corner of each panel. Scale bar, $200 \mu \mathrm{m}$

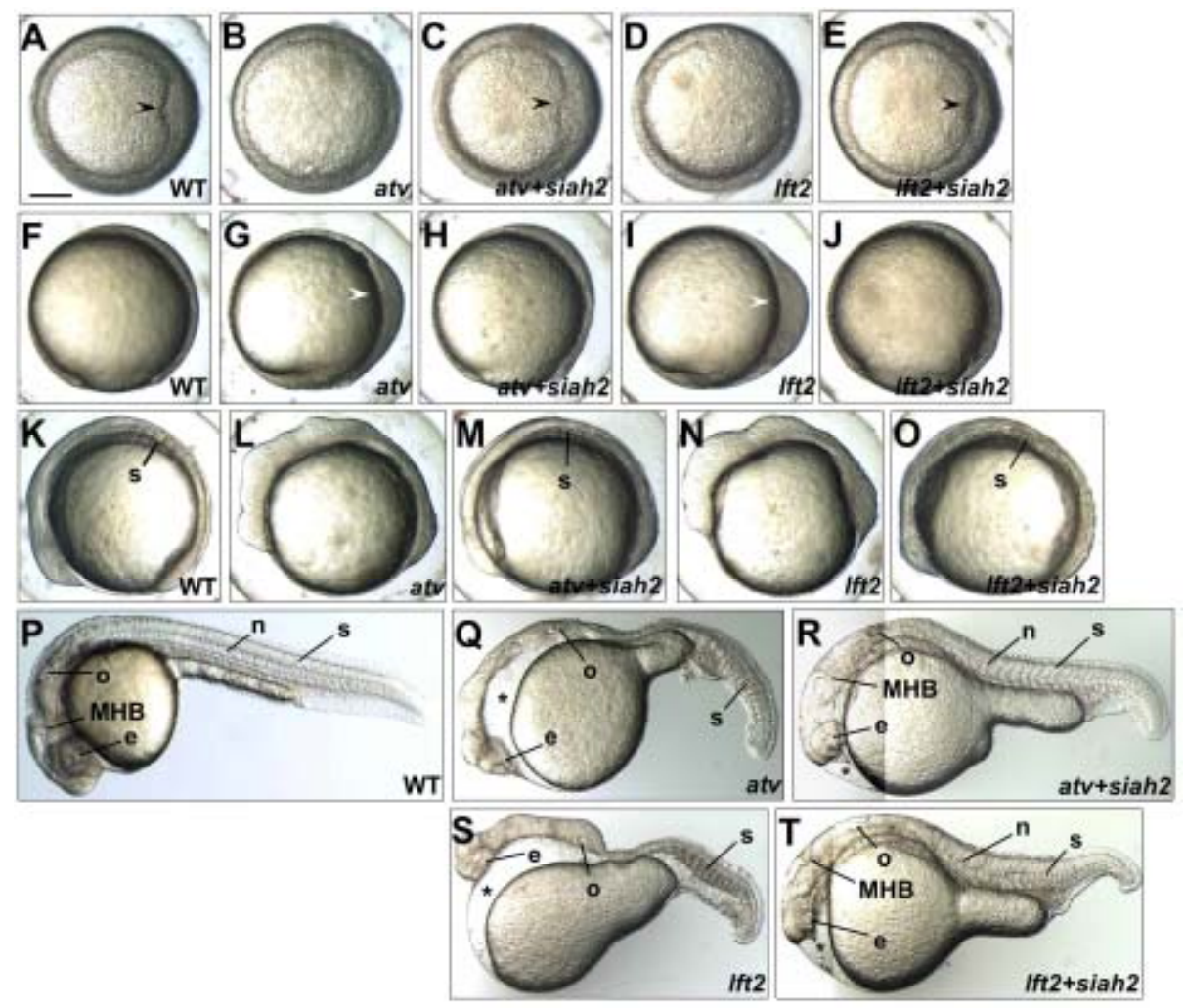

Fig. 4. Siah2 suppresses morphological defects induced by atv or Ift2 overexpression. (A, F, K, and P) WT control. Embryonic growth was examined throughout the developmental process. Embryonic shield marked by an arrowhead in $(A)$ was not formed in atv (25 pg) or Ift2 (25 $\mathrm{pg})$ injected embryos at $6 \mathrm{hpf}(\mathrm{B}, \mathrm{D})$. Shield structure was recovered by siah2 co-injection (100 pg) (arrowhead, C, E). At $90 \%$ epiboly, atv or Ift2 injected embryos accumulated cells on the dorsal side (arrowhead, G, I) while siah2 co-injected embryos did not show the dorsal thickening $(H, J)$. At 5 somites stage, the brain was enlarged and the somites were not visible in atv or Ift2 injected embryos (L, N). Co-injected with siah2 restored the brain and somites structures $(\mathrm{M}, \mathrm{O})$. atv or Ift2 injection induced cyclopia, lack of anterior mesendoderm, notochord and truck somites $(\mathrm{Q}, \mathrm{S})$. In the absence of notochord, somites with columnar shaped were fused under the neural tube $(\mathrm{Q}, \mathrm{S})$. Mesendodermal defects were partially restored by siah2 co-

injection $(R, T)$. Embryos are shown in the following orientations: (A-E) anterior view, dorsal to the right; (F-J) lateral view, dorsal to the right; (K-T) lateral view with anterior to the left. Abbreviations: s, somites; e, eye; o, otic vesicle; n, notochord. Scale bar, $200 \mu \mathrm{m}$.

which the Nodal signaling was experimentally compromised, we injected Nodal antagonist alone or together with siah2 followed by analyzing sqt and cyc expression. While atv/ft2 overexpression abolished sqt expression in the marginal blastomeres of late blastula (Figs. 6B and 6D), enforced siah2 expression overcame the atv or Ift2 mediated reduction of sqt expression (Figs. 6C and 6E). Additionally, compromised expression of cyc was also recovered at least in part by Siah2 (Figs. 6F-6J). The recovery of nodal related gene expression by Siah2 was measured further with RT-PCR (Fig. 6K). Taken together, our data suggested that Siah2 stimulates nodal re- lated genes expression in the marginal blastomere at late blastula through early gastrula, and the local stimulation of sqt and cyc expression in turn overcome inhibitory effects of atv/lft2, presumably by overriding competition against Nodal competitive inhibitors.

Fast1/FoxH1 is absolutely required for the Siah2 dependent Nodal augmentation

Because Nodal transduction pathway is mediated by intracellular Smad and FoxH1 (also called Fast1) protein complex (Attisano et al., 2001), we also examined whether Siah2 requires 


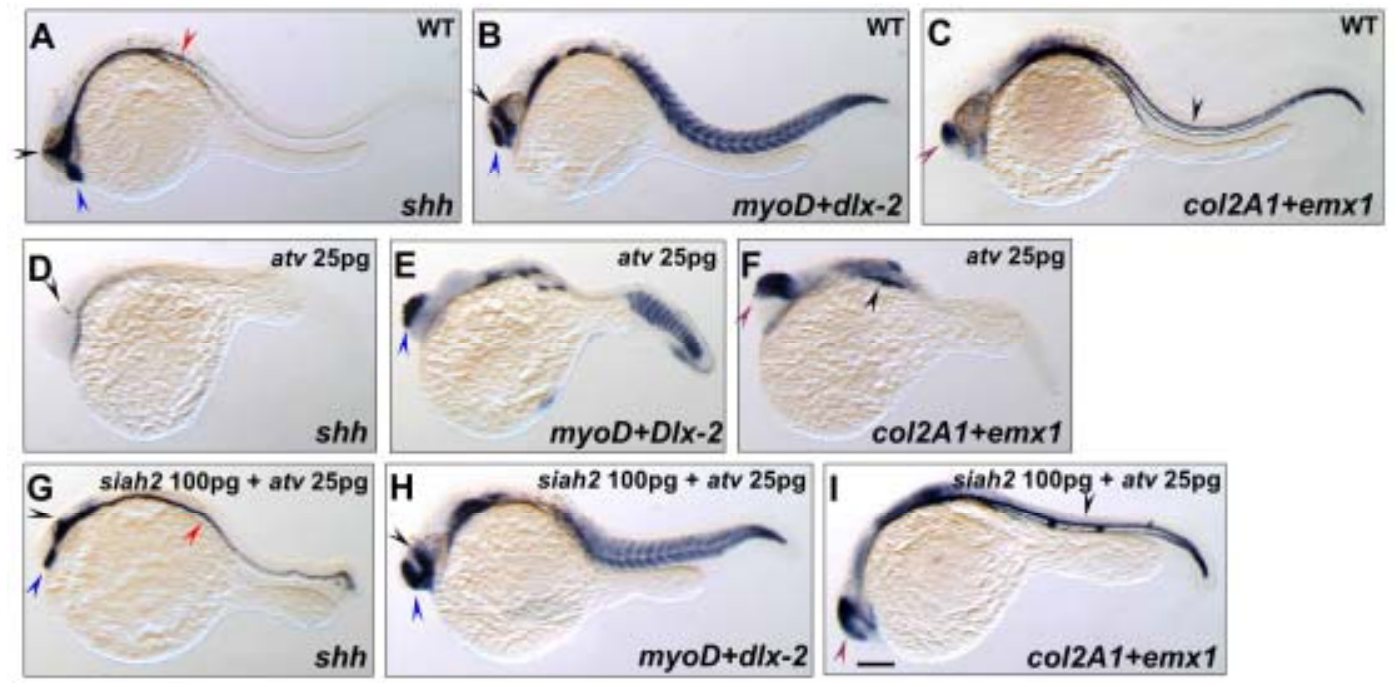

Fig. 5. Siah2 restored the embryonic defects induced by atv overexpression. (A, D, and G) Expression of shh in the ventral forebrain (blue arrowhead), anterior diencephalon (black arrowhead) and floor plate (red arrowhead) in WT embryos (A), but remarkably reduced in atv (25 $\mathrm{pg}$ ) injected embryo (D), shh expression was restored by siah2 co-expression (G). (B, E, and H) myoD expression in the somites (red arrowhead), $d l \times 2$ in the telencephalon (blue arrowhead) and anterior diencephalon (black arrowhead) of WT embryos (B). Pillar like expression of $m y o D$ was detected in the tail somites of atv injected embryos $(\mathrm{E})$. dlx2 expression in the telencephalon was enlarged at the expense of diencephalon (E). siah2 co-injection restored wedge like expression pattern of myoD in the trunk and dlx2 expression in the brain $(\mathrm{H})$. (C, F, and I) Expression of col2A1 in the notochord (red arrowhead) and emx1 in the telecephalon (blue arrowhead) of a control embryo (C). Absence of notochord and enlarged telencephalon was obvious in atv injected embryos (F). Siah2 overexpression rescued notochord and forebrain defects (I). All embryos were fixed at $24 \mathrm{hpf}$. Lateral view, anterior to the left. Scale bar, $200 \mu \mathrm{m}$.
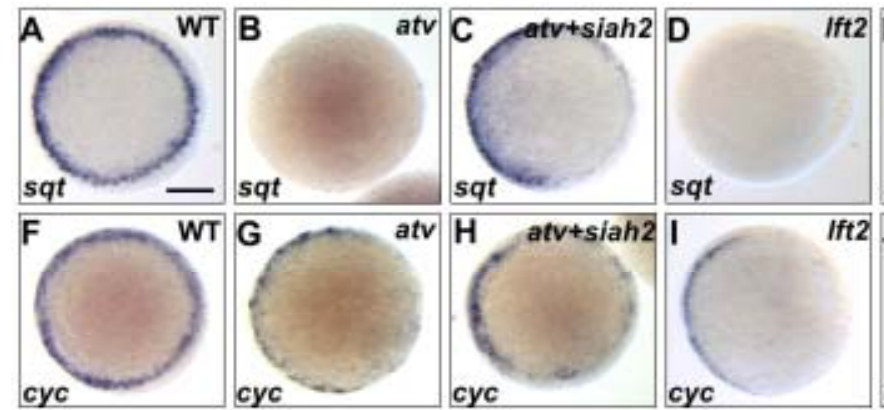

cyc

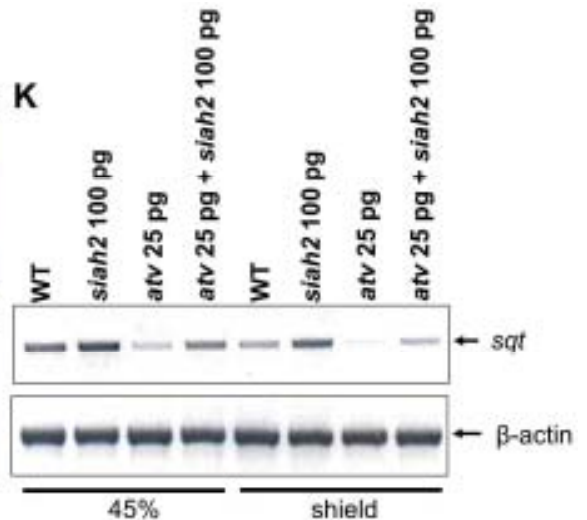

Fig. 6. Siah2 antagonizes the Atv or Lft2 activity. sqt (A-E) and cyc (F-J) transcripts were detected by in situ hybridization. (A) sqt expression was detected in the marginal blastomere of uninjected embryo at $45 \%$ epiboly stage. (B, D) sqt transcription was completely suppressed in atv or Ift2 RNA injected embryos. (C, E) siah2 co-injection restored the expression of sqt in the blastoderm margin. (F) cyc transcripts appeared in the marginal blastomeres of uninjected embryo at $45 \%$ epiboly. (G, I) atv or Ift2 RNA injection suppressed cyc expression from dorsal to the ventro-lateral margin. $(\mathrm{H}, \mathrm{J})$ Siah2 restored the cyc expression in the marginal blastomeres. $(\mathrm{K}) \mathrm{RT}$ PCR analysis with injected embryos at $45 \%$ epiboly and embryonic shield stage. Embryos were injected with RNA encoding siah2 $(100 \mathrm{pg})$ and/or atv $(25 \mathrm{pg})$. PCR (27 cycles) was carried out with primers as "Materials and Methods". $\beta$-actin PCR products were loaded as a loading control. (A-J) Animal pole view, dorsal to the right side. Scale bar, $200 \mu \mathrm{m}$.

the intracellular elements for its biological functions. We initially analyzed the effects of WT and dominant negative form of Fast1 on sqt transcripts. Embryos were injected with dominant negative fast1 (only containing Smad binding domain; fast1SID; $0.5 \mathrm{ng}$ ) (Pogoda et al., 2000) alone or together with siah2 (Fast1-SID; $0.5 \mathrm{ng}+$ siah2, $0.1 \mathrm{ng}$ ). Overexpression of fast1$S I D$ markedly reduced sqt transcripts in the marginal blastomere (9/20 lost, 10/20 showed reduced expression of sqt, Figs. $7 \mathrm{~A}$ and $7 \mathrm{~B}$ ) and developed the phenotype of MZsur, a maternal and zygotic mutant encoding two point mutations in forkhead domain of FoxH1 (data not shown) (Sirotkin et al., 2000). Embryos injected with fast1-SID and siah2 showed decreased or eliminated sqt expression in the blastoderm margin as well (10/22 lost, 10/22 reduced expression of sqt, Fig. 7C). However, Siah2 enhanced 3TP (containing 3 copy of Smad binding element of PIA1 gene)-Luc reporter gene activity (Fig. 7D) upon TGF- $\beta$ stimulation while Fast1-SID suppressed the reporter gene stimulated by Siah2 with TGF- $\beta$ treatment (Fig. 7D). 

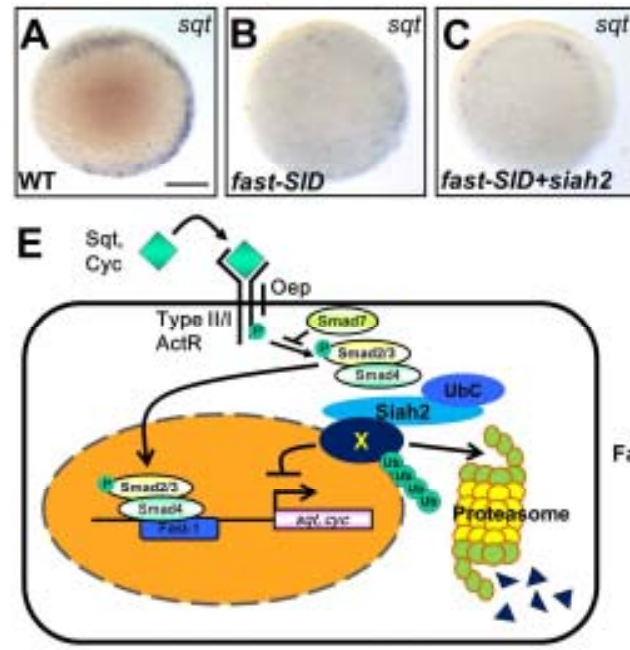

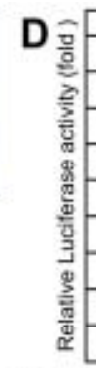

:

TGF- $\beta$

Siah2 Fast1-SID

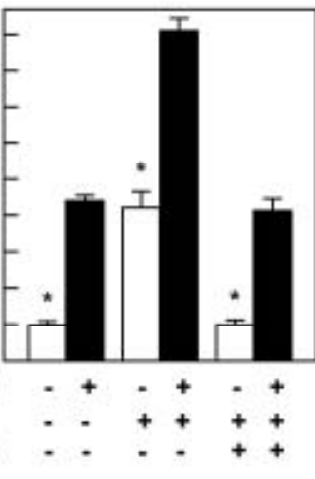

CFC/Oep co-receptor, which in turn activates type I activin like receptor. The activated type I receptor phosphorylates Smad2, and then the phosphorylated Smad2 binds with Smad4. The Smad2/4 translocates into nucleus to form a large transcriptional protein complex with Fast-1. The transcriptional complex re-activates nodal related genes through a positive feedback loop. We postulate that Siah2 (presumably together with Siah1) targets unidentified protein $\mathrm{X}$ which might serve as an intracellular Nodal signaling inhibitor for proteasome dependent protein degradation. *significance was taken as $\mathrm{P}<0.05$ Student's $t$-test. Scale bar, $200 \mu \mathrm{m}$.
A
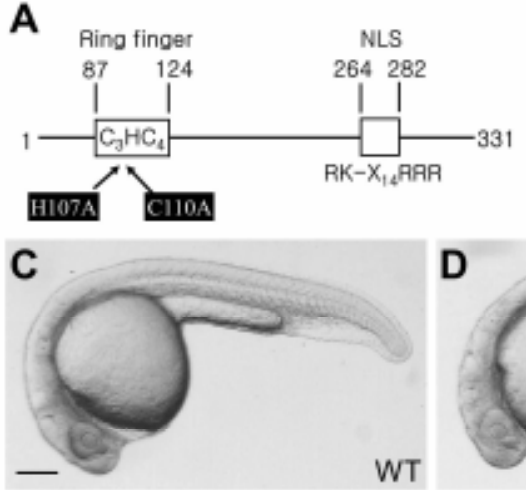

D

WT

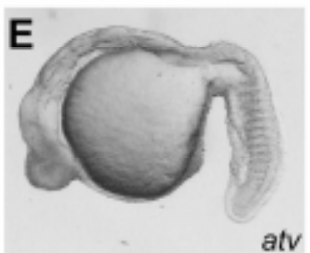

\section{$\mathbf{F}$}

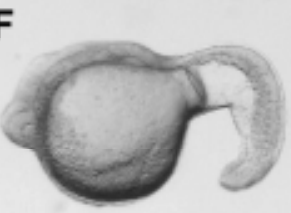

atv
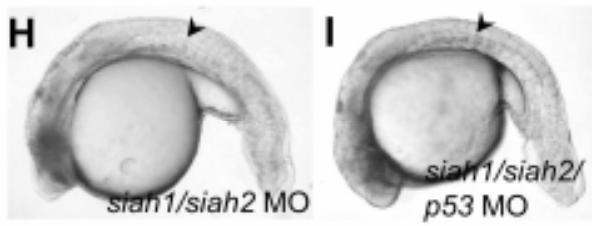

B

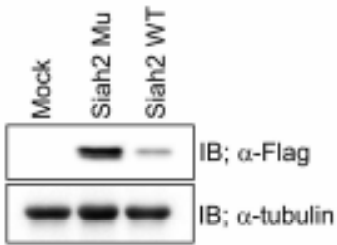

atv $+\operatorname{siah} 2 \mathrm{Mu}$
Fig. 8. E3 ubiquitin ligase activity of Siah2 is critical for antagonizing Antivin function. (A) Diagram represents RING mutated amino acid residues of Siah2. (B) Flag-tagged WT siah2 or RING mutated Siah2 (siah2 Mu) were transfected into 293T cell. Anti-Flag antibody was used to detect transfected WT Siah2 or Siah2 Mu (Top). The same membrane (middle) was stripped and blotted with $\alpha$-tubulin as a loading control (bottom). (C) WT embryo. (D) 200 pg siah2 Mu RNA injected embryo. (E) $25 \mathrm{pg}$ atv RNA injected embryo. (F) 25 pg atv RNA was co-injected with 200 pg siah2 Mu RNA. (C-F) All live embryos were taken picture at $24 \mathrm{hpf}$. Lateral view, anterior to the left. Arrowheads in $(\mathrm{H})$ and (I) indicate notochord. Scale bar, 200 $\mu \mathrm{m}$.
These studies suggest that Siah2 requires Fast1 transcription factor to stimulate transcription of Nodal downstream genes, such as nodal related genes themselves. Because the Siah2 dependent 3TP-Luc reporter gene activity was additive to TGF$\beta$ stimuli (Fig. 7D), we propose that Siah2 may exert its biological function in TGF- $\beta$ independent manner. It is thus conceivable that TGF- $\beta$ and Siah2 mediated gene induction pathways merge into Fast1/FoxH1, and that Siah2 may destabilize unidentified intracellular TGF- $\beta /$ Nodal inhibitor(s) by exploiting ubiquitin proteasome system (UPS) (Fig. 7E).

E3 ubiquitin ligase activity of Siah2 is required for Nodal signaling

Siah proteins bind E2 ubiquitin conjugating enzymes via N- 
terminal RING domain and interact with proteins targeted for degradation via C-terminal domain (Wong and Möller, 2013). Given that nodal related gene expression was augmented by Siah2 overexpression, we introduced two point mutations into the $\mathrm{C}_{3} \mathrm{HC}_{4}$ zinc RING finger domain by substituting core histidine and cysteine residues with alanine (H107A, C110A) (Siah $\mathrm{Mu}$ ) to eliminate Siah2 E3 ubiquitin ligase activity (Fig. 8A). Flag tagged WT siah2 or siah Mu were transfected into the 293T mammalian cells, of which the cellular extracts were subject to immunoblotting. As shown in Fig. 8B, the increased stability of Siah2 by inducing mutations indicated that E3 ubiquitin ligase activity was inactivated in Siah Mu. Embryos overexpressing Siah Mu developed overall WT-like morphology with certain degree of cell growth retardation and shortened body size (Fig 8D). When Siah Mu was co-expressed with Antivin, Siah Mu was unable to rescue the phenotypic defects induced by Nodal blockage (Figs. 8E-8G). These results clearly argue that E3 ubiquitin ligase activity of Siah2 is essential for transducing proper Nodal signaling.

Siah depleted embryos partly mimics Nodal defective phenotype

To explore the in vivo function of Siah during early embryonic development, we depleted both Siah1 and Siah2 using antisense morpholinos (MO). As shown in Figs. 8H and 8l, Siah depleted embryos exhibited severe body defects with shorten axis and defected mesodermal tissues somewhat similar to the embryos with compromised Nodal signaling. It is noteworthy that since the only tissue necrosis presumably due to siah $\mathrm{MO}$ toxicity was significantly ameliorated by inhibiting p53 function without any improved body structure of the morphants, we concluded that the embryonic defects induced by depletion of Siah1 and Siah2 were not simply due to the MO toxicity but mainly due to the compromised Siah function during early embryonic development. However, a rudimentary notochord was still observed in siah morphants, and the columnar shape of somites, which is the another hallmark of Nodal deficiency, was less obvious in siah morphants. In addition, contrary to our expectations, siah morphants showed equivalent expression of sqt and cyc at $40 \%$ epiboly to WT (data not shown). There are at least two possible explanations for the unexpected results. First, the maternally deposited Siah deviated from MO targeting might compensate the early induction of mesendodermal cells in zebrafish embryos. Second, the Siah might be less permissive but more instructive to the Nodal signaling during early embryonic development, even if compound Siah1a and Siah2 mutant mice showed neonatal lethality without any overt histological defects (Frew et al., 2003). Therefore, generation of Siah1a, Siah1b and Siah2 triple compound mutant mice will be necessary to identify whether the Siah dependent Nodal signaling is conserved across species. Nevertheless, the defective mesendoderm derived tissues in the siah morphants argues that Nodal signaling is positively influenced by endogenously expressed Siah.

\section{DISCUSSION}

Highly conserved Siah homologues have been identified in fly, mouse, zebrafish and human. Here we reported that Siah augmented a sufficient quantity of nodal related genes expression in the marginal blastomeres, which is enough to override the competition against the biological functions of Nodal antagonists (Atv and Lft). Given the reported E3 ubiquitin ligase activities of Siah family and the versatile physiological function of UPS system (Anuppalle et al., 2013; Qi et al., 2013), it is worth consideration that Siah may target intracellular inhibitor(s) of Nodal signaling for proteasome dependent protein destruction to increase the duration or magnitude of the expression of nodal related genes. Although several Siah target proteins involved in diverse cellular signaling pathways have been identified (House et al., 2009), there are lack of any in vivo evidences for the physiological function of Siah in TGF- $\beta$ or Nodal signaling pathway.

One of the plausible targets of Siah in Nodal signaling is TIEG1, a transcriptional repressor of smad7 promoter, which can be targeted for destruction by Siah (Jonhson et al., 2002). Thus, TIEG1 enhances TGF- $\beta /$ Smad signaling by inhibiting negative feedback regulation loop mediated by inhibitory Smad7 (Johnsen et al., 2002). Given when Smad7 was overexpressed in zebrafish, the embryos showed dorsalized or ventralized, sometimes both combined phenotype due to the binary inhibition of Nodal and BMP signaling by Smad7 (Pogoda and Meyer, 2002), which is reminiscent of the embryos with elevated Siah2 expression by mRNA injection (Fig. 1). In this context, we tested if Siah2 stimulates smad7 expression in the marginal blastomeres by down regulating TIEG1 protein stability. However, siah1 or siah2 mRNA injection failed to expand smad7 expression level during early to mid-gastrula stage (data not shown). This observation may be explained by the fact that, in the absence of growth factor, TIEG1 is scarcely expressed at levels which could not show any biological activity (Johnsen et al., 2002). Thus, we concluded that TIEG1 may not be the major target of Siah for the maintenance of proper Nodal signaling, even if we cannot exclude the possibility that the relatively low cellular level of TIEG1 regulated by Siah somewhat contributes to the maintenance of Nodal homeostasis.

Another putative target of Siah in Nodal signaling is Zic2. Houston and Wylie (2005) showed that maternally supplied Zic2 suppressed the expression of several Xenopus nodal related $(X n r)$ genes in early development. Depletion of maternally expressed Zic2 in Xenopus embryos resulted in exogastrulation, anterior truncation and axial defect. In addition, loss of maternal Zic2 caused increased expression of $\mathrm{Xnr}$ genes, and hyper-activation of Nodal signaling. However, we failed to detect physical association between Siah2 and Zic2 (data not shown). Since Zic2 was not poly-ubiquitylated and not destabilized by Siah, we ruled out Zic2 as a target molecule of Siah.

Iratni et al. (2002) reported that transcriptional corepressor DRAP1 interacts with Fast-1 and inhibits DNA binding. Interestingly, the DRAP1-deficient mice showed expanded Nodal expression in primitive streak, while the lft2 transcription was largely diminished in nascent mesoderm (Iratni et al., 2002). Therefore, DRAP1 was one of the attractive candidates of Siah. However, DRAP1 does not contain well conserved Siah degron sequence (RPVAxVxPxxR; VxP is the core Siah binding motif) (House et al., 2003; Möller et al., 2009) across the species and may not physically interact with Siah2.

We previously identified a novel Siah2 interacting partner designated as Sinup using yeast two-hybrid screening with Siah2 as bait (Ro et al., 2005). Overexpression of sinup induced cyclopia-like phenotype, reminiscent of the Zeop mutant, but did not affect the mRNA level of sqt and cyc (Ro et al., 2005). Thus, we concluded that the fused-eye phenotype induced by Sinup overexpression was not due to the attenuated Nodal signaling by the elevated level of Sinup, but simply due to the anterior neural plate defects.

A previous report showed that T84 epithelial cells treated with TGF- $\beta$ down regulates Siah1 and Siah2 expression level when 
the cells were cultured three-dimensionally (Juuti-Uusitalo et al., 2006). Recently, Liao et al. (2012) also reported that undifferentiated small intestinal crypt cells (IEC-6 cell line) in response to TGF- $\beta$ stimuli increased the expression of miRNA-146b, which induced $\mathrm{SIAH} 2 \mathrm{mRNA}$ decay presumably through direct interaction between the seed region of miRNA-146b and its complementary sequences of SIAH2 3'UTR. Although it is not clear whether the TGF- $\beta$ dependent Siah regulatory loop is generic feedback mechanism, the previous reports (Juuti-Uusitalo et al., 2006; Liao et al., 2012) suggested that nodal related gene expression can be negatively regulated by Nodal itself through suppressing Siah expression, which rendered the further complexity of the regulatory loop of Nodal signaling. However, at least in zebrafish, since the Siah1 and Siah2 are largely accumulated as maternal transcripts, the maternally deposited siah transcripts may contribute the onset as well as maintenance of nodal related genes expression during late blastula and early gastrula stage. Collectively to our knowledge, this is the first report unveiling hidden utility and function of Siah on the way to augment nodal related genes expression for the maintenance of Nodal homeostasis. Obviously, identification of target proteins of Siah in Nodal signaling will extend our understanding molecular paradigm governing vertebrate embryogenesis.

\section{ACKNOWLEDGMENTS}

We thank I.B. Dawid (NIH-NICHD, USA) for the valuable comments for and thorough reading of our manuscript. This research was supported by Basic Science Research Program through the National Research Foundation of Korea (NRF) funded by the Ministry of Education, Science and Technology (NRF-2012R1A1A4A01012264).

\section{REFERENCES}

Anuppalle, M., Maddirevula, S., Huh, T.R., and Rhee, M. (2013). Ubiquitin proteasome system networks in the neurological disorder. Anim. Cells Syst. 17, 383-387.

Attisano, L., Silvestri, C., Izzi, L., and Labbe, E. (2001). The transcriptional role of Smads and FAST (FoxH1) in TGF- $\beta$ and activin signalling. Mol. Cell. Endocrinol. 180, 3-11.

Bisgrove, B.W., Essner, J.J., and Yost, H.J. (1999). Regulation of midline development by antagonism of lefty and nodal signaling. Development 236, 3253-3262.

Carthew, R.W., and Rubin, G.M. (1990). seven in absentia, a gene required for specification of $\mathrm{R} 7$ cell fate in the Drosophila eye. Cell 63, 561-577.

Chen, X., Weisberg, E., Fridmacher, V., Watanabe, M., Naco, G., and Whitman, M. (1997). Smad4 and FAST-1 in the assembly of activin-responsive factor. 389, 85-89.

Erter, C.E., Solnica-Krezel, L., and Wright, C.V. (1998). Zebrafish nodal-related 2 encodes an early mesendodermal inducer sig naling from the extraembryonic yolk syncytial layer. Dev. Biol. 204, 361-372.

Erter, C.E., Wilm, T.P., Basler, N., Wright, C.V., and Solnica-Krezel, L. (2001). Wnt8 is required in lateral mesendodermal precursors for neural posteriorization in vivo. Development 128, 3571-3583.

Feldman, B., Gates, M.A., Egan, E.S., Dougan, S.T., Rennebeck, G., Sirotkin, H.I., Schier, A.F., and Talbot, W.S. (1998). Zebrafish organizer development and germ-layer formation require nodal-related signals. Nature 395, 181-185.

Frew, I.J., Hammond, V.E., Dickins, R.A., Quinn, J.M., Walkley, C.R., Sims, N.A., Schnall, R., Della, N.G., Holloway, A.J., Digby, M.R., et al. (2003). Generation and analysis of Siah2 mutant mice. Mol. Cell. Biol. 23, 9150-9161.

Gore, A.V., Maegawa, A., Cheong, A., Gilligan, P.C., Weinberg, E.S., and Sampath, K. (2005). The zebrafish dorsal axis is apparent at the four-cell stage. Nature 438, 1030-1035.

Gritsman, K., Zhang, J., Cheng, S., Heckscher, E., Talbot, W.S., and Schier, A.F. (1999). The EGF-CFC protein one-eyed pinhead is essential for nodal signaling. Cell 97, 121-132.
House, CM., Frew, I.J., Huang, H.L., Wiche, G., Traficante, N., Nice, E., Catimel, B., and Bowtell, D.D. (2003). A binding motif for Siah ubiquitin ligase. Proc. Natl. Acad. Sci. USA 100, 3101-3106.

House, C.M., Möller, A., and Bowtell, D.L. (2009). Siah protein: novel drug targets in the ras and hypoxia pathways. Cancer Res. 69, 8835-8838.

Houston, D.W., and Wylie, C. (2005). Maternal Xenopus Zic2 negatively regulates Nodal-related gene expression during anteroposterior patterning. Development 132, 4845-4855.

Hu, G., Chung, Y.L., Glover, T., Valentine, V., Look, A.T., and Fearon, E.R. (1997). Characterization of human homologs of the Drosophila seven in absentia (sina) gene. Genomics 46, 103111.

Huelsken, J., and Birchmeier, W. (2001). New aspects of Wnt signaling pathways in higher vertebrates. Curr. Opin. Genet. Dev. 11, 547-553.

Iratni, R., Yan, Y.T., Chen, C., Ding, J., Zhang, Y., Price, S.M., Reinberg, D., and Shen, M.M. (2002). Inhibition of excess nodal signaling during mouse gastrulation by the transcriptional corepressor DRAP1. Science 298, 1996-1999.

Johnsen, S.A., Subramaniam, M., Monroe, D.G., Janknecht, R. and Spelsberg, T.C. (2002). Modulation of transforming growth factor (TGF- $\beta$ )/Smad transcriptional responses through targeted degradation of TGF- $\beta$-inducible early gene- 1 by human seven in absentia homologue. J. Biol. Chem. 277, 30754-30759.

Juuti-Uusitalo, K.M., Kaukinen, K., Mäki, M., Tuimala, J., and Kainulainen, H. (2006). Gene expression in TGFbeta-induced epithelial cell differentiation in a three-dimensional intestinal epithelial cell differentiation model. BMC Genomics 7, 279.

Langdon, Y.G., and Mullins, M.C. (2011). Maternal and zygotic control of zebrafish dorsoventral axial patterning. Annu. Rev. Genet. 45, 357-377.

Li, S., Li, Y., Carthew, R.W., and Lai, Z.C. (1997). Photoreceptor cell differentiation requires regulated proteolysis of the transcriptional repressor tramtrack. Cell 90, 469-478.

Liao, Y., Zhang, M., and Lönnerdal, B. (2012). Growth factor TGF- $\beta$ induces intestinal epithelial cell (IEC-6) differentiation: miR-146b as a regulatory component in the negative feedback loop. Genes Nutr. 8, 69-78.

Liu, J., Stevens, J., Rote, C.A., Yost, H.J., Hu, Y., Neufeld, K.L. White, R.L., and Matsunami, N. (2001). Siah-1 mediates a nove $\beta$-catenin degradation pathway linking p53 to the adenomatous polyposis coli protein. Mol. Cell 7, 927-936.

Lu, F.I., Thisse, C., and Thisse, B. (2011). Identification and mechanism of regulation of the zebrafish dorsal determinant. Proc Natl. Acad. Sci. USA 108, 15876-15880.

Matsuzawa, S., and Reed, J.C. (2001). Siah-1, SIP, and Ebi collaborate in a novel pathway for b-catenin degradation linked to p53 responses. Mol. Cell 7, 915-926.

Matsuzawa, S., Takayama, S., Froesch, B.A., Zapata, J.M., and Reed, J.C. (1998). p53-inducible human homologue of Drosophila seven in absentia (Siah) inhibits cell growth: suppression by BAG-1. EMBO J. 17, 2736-2747.

Medhioub, M., Vaury, C., Hamelin, R., and Thomas, G. (2000). Lack of somatic mutation in the coding sequence of SIAH1 in tumors hemizygous for this candidate tumor suppressor gene. Int. J. Cancer 87, 794-797.

Möller, A., House, C.M., Wong, C.S., Scanlon, D.B., Liu, M.C., Ronai, Z., and Bowtell, D.D. (2009). Inhibition of Siah ubiquitin ligase function. Oncogene 28, 289-296.

Nadeau, R.J., Toher, J.L., Yang, X., Kovalenko, D., and Friesel, R. (2006). Regulation of Sprouty2 stability by mammalian Sevenin-Absentia homolog 2. J. Cell Biochem. 100, 151-160.

Pogoda, H.M., and Meyer, D. (2002). Zebrafish Smad7 is regulated by Smad3 and BMP signals. Dev. Dyn. 224, 334-349.

Pogoda, H.M., Solnica-Krezel, L., Driever, W., and Meyer, D. (2000). The zebrafish forkhead transcription factor FoxH1/Fast1 is a modulator of nodal signaling required for organizer formation. Curr. Biol. 10, 1041-1049.

Qi, J., Nakayama, K., Gaitonde, S., Goydos, J.S., Krajewski, S. Eroshkin, A., Bar-Sagi, D., Bowtell, D., and Ronai, Z. (2008). The ubiquitin ligase Siah2 regulates tumorigenesis and metastasis by HIF-dependent and -independent pathways. Proc. Natl. Acad. Sci. USA 105, 16713-16718.

Qi, J., Kim, H., Scortegagna, M., and Ronai, Z.A. (2013). Regulators and effectors of Siah ubiquitin ligases. Cell Biochem. Bio- 
phys. $67,15-24$

Rebagliati, M.R., Toyama, R., Haffter, P., and Dawid, I.B. (1998). cyclops encodes a nodal-related factor involved in midline signaling. Proc. Natl. Acad. Sci. USA 95, 9932-9937.

Ro, H., Kim, K.E., Huh, T.L., Lee, S.-K., and Rhee, M. (2003). Expression pattern of Siaz gene during the zebrafish embryonic development. Gene Exp. Patterns 3, 483-488.

Ro, H., Jang, Y., and Rhee, M. (2004a). The ring domain of Siaz, the zebrafish homologue of Drosophila seven in absentia, is essential for cellular growth arrest. Mol. Cells 17, 160-165.

Ro, H., Soun, K., Kim, E-J., and Rhee, M. (2004b). Novel vector systems optimized for injecting in vitro-synthesized mRNA into zebrafish embryos. Mol. Cells 17, 373-376.

Ro, H., Won, M., Lee, S.U., Kim, K.E., Huh, H.L., Kim, C.H., and Rhee, M. (2005). Sinup, a novel Siaz-interacting nuclear protein, modulates neural plate formation in the zebrafish embryos. Biochem. Biophys. Res. Commun. 332, 993-1003.

Robu, M.E., Larson, J.D., Nasevicius, A., Beiraghi, S., Brenner, C., Farber, S.A., and Ekker, S.C. (2007). p53 activation by knockdown technologies. PLoS Genet. 3, e78.

Schier, A.F., and Talbot, W.S. (2001). Nodal signaling and the zebrafish organizer. Int. J. Dev. Biol. 45, 289-297.
Schier, A.F., and Talbot, W.S. (2005). Molecular genetics of axis formation in zebrafish. Annu. Rev. Genet. 39, 561-613.

Sirotkin, H.I., Gates, M.A., Kelly, P.D., Schier, A.F., and Talbot, W.S. (2000). Fast1 is required for the development of dorsal axial structures in zebrafish. Curr. Biol. 10, 1051-1054.

Tang, A.H., Neufeld, T.P., Kwan, E., and Rubin, G.M. (1997). PHYL acts to down-regulate TTK88, a transcriptional repressor of neuronal cell fates, by a SINA-dependent mechanism. Cell 90, 459467.

Thisse, C., and Thisse, B. (1999). Antivin, a novel and divergent member of the TGF- $\beta$ superfamily, negatively regulates mesoderm induction. Development 126, 229-240.

Thisse, B., Wright, C.V., and Thisse, C. (2000). Activin- and Nodalrelated factors control antero-posterior patterning of the zebrafish embryo. Nature 403, 425-428.

Westerfield, M. (1995). The Zebrafish book: a guide for the laboratory use of zebrafish (Danio rerio) (University of Oregon Press).

Whitman, M. (2001). Nodal signaling in early vertebrate embryos: themes and variations. Dev. Cell 1, 605-617.

Wong, C.S., and Möller, A. (2013). Siah: a promising anticancer target. Cancer Res. 73, 2400-2406. 